\title{
HOMOGENIZATION OF FUČÍK EIGENCURVES
}

\author{
JULIÁN FERNÁNDEZ BONDER, JUAN PABLO PINASCO AND ARIEL MARTIN SALORT
}

\begin{abstract}
In this work we study the convergence of an homogenization problem for half-eigenvalues and Fučík eigencurves. We provide quantitative bounds on the rate of convergence of the curves for periodic homogenization problems.
\end{abstract}

\section{INTRODUCTION}

Given a bounded interval $(0, \ell) \subset \mathbb{R}$, we are interested in the asymptotic behavior, as $\varepsilon \rightarrow 0$, of the spectrum of the following family of asymmetric elliptic problems

$$
\left\{\begin{array}{l}
-u^{\prime \prime}=\alpha m_{\varepsilon}(x) u^{+}-\beta n_{\varepsilon}(x) u^{-}, \quad x \in(0, \ell) \\
u(0)=u(\ell)=0,
\end{array}\right.
$$

where $(\alpha, \beta) \in \mathbb{R}_{+}^{2}$, and the functions $m_{\varepsilon}, n_{\varepsilon} \in L^{\infty}([0, \ell])$ are positive and uniformly bounded between two positive constants,

$$
0<a \leq m_{\varepsilon}(x), n_{\varepsilon}(x) \leq b<\infty .
$$

As usual, given a function $u$ we denote by $u^{ \pm}=\max \{0, \pm u\}$ the positive and negative parts of $u$.

Here we assume that there exist functions $m_{0}, n_{0}$ such that

$$
m_{\varepsilon} \stackrel{*}{\rightarrow} m_{0} \quad \text { and } \quad n_{\varepsilon} \stackrel{*}{\rightarrow} n_{0} \quad \text { weakly* in } L^{\infty}([0, \ell]) \text {. }
$$

It is well-known that in the case of periodic homogenization, where

$$
m_{\varepsilon}(x)=m\left(\frac{x}{\varepsilon}\right), \quad n_{\varepsilon}(x)=n\left(\frac{x}{\varepsilon}\right)
$$

for some $\ell$-periodic functions $m, n \in L^{\infty}(\mathbb{R})$, we have that $m_{\varepsilon} \stackrel{*}{\rightarrow} m_{0}$ and $n_{\varepsilon} \stackrel{*}{\rightarrow} n_{0}$ as $\varepsilon \rightarrow 0$, where

$$
m_{0}=\bar{m}:=\frac{1}{\ell} \int_{0}^{\ell} m(x) d x \quad \text { and } \quad n_{0}=\bar{n}:=\frac{1}{\ell} \int_{0}^{\ell} n(x) d x .
$$

We will show that the following limit equation is obtained,

$$
\left\{\begin{array}{l}
-u^{\prime \prime}=\alpha m_{0} u^{+}-\beta n_{0} u^{-} \quad x \in(0, \ell) \\
u(0)=u(\ell)=0,
\end{array}\right.
$$

in the sense that, from any sequence of weak solutions $\left\{\left(\alpha_{\varepsilon_{j}}, \beta_{\varepsilon_{j}}, u_{\varepsilon_{j}}\right)\right\}_{j \geq 1}$ of $\left(P_{\varepsilon}\right)$, with $\varepsilon_{j} \rightarrow 0$, uniformly bounded in $\mathbb{R}^{2} \times H_{0}^{1}([0, \ell])$, we can extract a strongly convergent subsequence in $\mathbb{R}^{2} \times L^{2}([0, \ell])$, weakly convergent in $\mathbb{R}^{2} \times H_{0}^{1}([0, \ell])$, and

2010 Mathematics Subject Classification. 35B27, 35P15, 35P30.

Key words and phrases. Fučík eigenvalues, homogenization, order of convergence. 
the limit is a weak solution of equation $\left(P_{0}\right)$. Here, by a weak solution of $\left(P_{\varepsilon}\right)$ with $\varepsilon \geq 0$, we understand a pair $(\alpha, \beta) \in \mathbb{R}_{+}^{2}$ and $u \in H_{0}^{1}([0, \ell])$ satisfying

$$
\int_{0}^{\ell} u^{\prime} v^{\prime} d x=\int_{0}^{\ell}\left(\alpha m_{\varepsilon} u^{+}-\beta n_{\varepsilon} u^{-}\right) v d x
$$

for any $v \in H_{0}^{1}([0, \ell])$.

In order to study the convergence of the spectra of the problems $\left(P_{\varepsilon}\right)$ to the spectrum of $\left(P_{0}\right)$, let us recall some known facts about the structure of the socalled Fučík spectrum, introduced in the '70s by Dancer and Fučík, see $[4,7]$. For any $\varepsilon \geq 0$ fixed, let us denote by

(1.3) $\Sigma_{\varepsilon}=\Sigma\left(m_{\varepsilon}, n_{\varepsilon}\right):=\left\{(\alpha, \beta) \subset \mathbb{R}^{2}\right.$ : there exists a nontrivial solution of $\left.\left(P_{\varepsilon}\right)\right\}$,

the Fučík spectrum of problem $\left(P_{\varepsilon}\right)$. In the above mentioned references, it is proved that the spectrum $\Sigma_{\varepsilon}$ has the structure

$$
\Sigma_{\varepsilon}=\mathcal{C}_{0, \varepsilon}^{ \pm} \cup \bigcup_{k \in \mathbb{N}} \mathcal{C}_{k, \varepsilon}^{ \pm}
$$

where each $\mathcal{C}_{k, \varepsilon}^{ \pm}$is a curve in $\mathbb{R}^{2}$ for any $k \geq 0$. The curves $\mathcal{C}_{0, \varepsilon}^{ \pm}$are called the trivial curves and are given by $\mathcal{C}_{0, \varepsilon}^{+}=\left\{\lambda_{1}^{m_{\varepsilon}}\right\} \times \mathbb{R}, \mathcal{C}_{0, \varepsilon}^{-}=\mathbb{R} \times\left\{\lambda_{1}^{n_{\varepsilon}}\right\}$, where $\lambda_{k}^{r}$ denotes the $k$-th eigenvalue of the Dirichlet laplacian in $(0, \ell)$ with weight $r \in L^{\infty}([0, \ell])$, namely

$$
\left\{\begin{array}{l}
-u^{\prime \prime}=\lambda r(x) u, \quad x \in(0, \ell) \\
u(0)=u(\ell)=0 .
\end{array}\right.
$$

Observe that any eigenfunction associated to $\lambda_{1}^{r}$ has constant sign.

The curves $\mathcal{C}_{k, \varepsilon}^{+}$(resp. $\mathcal{C}_{k, \varepsilon}^{-}$) with $k \geq 1$ correspond to nontrivial solutions having $k$ internal zeros and positive (resp. negative) slope at the origin.

We have two curves for every $k \in \mathbb{N}$. In the constant coefficient case, for $k$ even, both curves coincide but this is not true for general weights.

The curves $\mathcal{C}_{k, \varepsilon}^{ \pm}$are not known explicitly for general weights $m_{\varepsilon}, n_{\varepsilon}$, and only its asymptotic behavior as $\alpha \rightarrow \infty$ (or $\beta \rightarrow \infty$ ) is known, see $[15,16]$.

The study of homogenization problems for asymmetric eigenvalues is not well understood nowadays. We cite the paper [13] of Malik where the homogenization problem for a model of suspension bridges was studied. In that work the author studies a model where the cable resist the expansion but does not resist compression. More recently, in [12], Li and Yan studied the continuity of the eigenvalues $\lambda\left(a_{n}, b_{n}\right)$ of the problem

$$
-\left(\left|u^{\prime}\right|^{p-2} u^{\prime}\right)^{\prime}=\lambda|u|^{p-2} u+a_{n}(x)\left|u^{+}\right|^{p-2} u^{+}-b_{n}(x)\left|u^{-}\right|^{p-2} u^{-}, \quad x \in(0, \ell)
$$

with homogeneous boundary conditions

$$
c_{11} u(0)+c_{12} u^{\prime}(0)=0=c_{21} u(\ell)+c_{22} u^{\prime}(\ell),
$$

and the convergence to the eigenvalues of

$$
-\left(\left|u^{\prime}\right|^{p-2} u^{\prime}\right)^{\prime}=\lambda|u|^{p-2} u+a(x)\left|u^{+}\right|^{p-2} u^{+}-b(x)\left|u^{-}\right|^{p-2} u^{-}, \quad x \in(0, \ell)
$$

with the same boundary conditions, where $a_{n} \rightarrow a$ and $b_{n} \rightarrow b$ weakly in $L^{\gamma}([0, \ell])$ for $1 \leq \gamma<\infty$. 
Also, the behavior as $\varepsilon \rightarrow 0$ of the first nontrivial curve in the Fučík spectrum for the $p$-Laplace operator in $\mathbb{R}^{n}$ for $n \geq 1$ was obtained by the third author in [17].

On the other hand, the homogenization of spectral problems in the symmetric case have been widely studied in both the linear and quasilinear cases. See for example $[1,2,3,5,8,9,10,14]$ and the references therein.

In this work we prove the convergence of the eigenvalues of problem $\left(P_{\varepsilon}\right)$ to the ones of problem $\left(P_{0}\right)$. Moreover, in the case of periodic homogenization we obtain the rate of convergence whenever we restrict $\Sigma_{\varepsilon}$ and $\Sigma_{0}$ to a line through the origin, and we give explicit bounds depending on $\varepsilon, k$, and the slope of the line.

Since the constant degenerates when the line approaches the axis, it is convenient to denote, for any $0<t<1$ by $\mathcal{K}_{t}$ a symmetric cone in the first quadrant defined by

$$
\mathcal{K}_{t}:=\left\{(\alpha, \beta) \in \mathbb{R}_{+} \times \mathbb{R}_{+}: t \alpha \leq \beta \leq t^{-1} \alpha\right\} .
$$

Our main results are the following:

Theorem 1.1 (General convergence). Let $\left\{m_{\varepsilon}\right\}_{\varepsilon>0}$ and $\left\{n_{\varepsilon}\right\}_{\varepsilon>0}$ be two families of weights satisfying (1.1) such that

$$
m_{\varepsilon} \stackrel{*}{\rightarrow} m_{0} \quad \text { and } \quad n_{\varepsilon} \stackrel{*}{\rightarrow} n_{0}
$$

weakly* in $L^{\infty}([0, \ell])$ and let $\Sigma_{\varepsilon}$ be the associated Fučík spectrum defined in (1.3).

Let $\left(\alpha_{k, \varepsilon}, \beta_{k, \varepsilon}\right) \in \mathcal{C}_{k, \varepsilon} \cap \mathcal{K}_{t} \subset \Sigma_{\varepsilon}$. Then, $\left\{\left(\alpha_{k, \varepsilon}, \beta_{k, \varepsilon}\right)\right\}_{\varepsilon>0}$ is bounded in $\mathbb{R}^{2}$ and if $\left(\alpha_{k, 0}, \beta_{k, 0}\right)$ is any accumulation point of $\left\{\left(\alpha_{k, \varepsilon}, \beta_{k, \varepsilon}\right)\right\}_{\varepsilon>0}$, then $\left(\alpha_{k, 0}, \beta_{k, 0}\right) \in$ $\mathcal{C}_{k, 0} \cap \mathcal{K}_{t} \subset \Sigma_{0}$.

Moreover, if $\left(\alpha_{k, \varepsilon}, \beta_{k, \varepsilon}\right) \in \mathcal{C}_{k, \varepsilon}^{+} \cap \mathcal{K}_{t}$, then $\left(\alpha_{k, 0}, \beta_{k, 0}\right) \in \mathcal{C}_{k, 0}^{+}$and analogous result for $\mathcal{C}_{k, \varepsilon}^{-}$.

Finally, if $u_{\varepsilon} \in H_{0}^{1}([0, \ell])$ is an eigenfunction of $\left(P_{\varepsilon}\right)$ associated to $\left(\alpha_{\varepsilon}, \beta_{\varepsilon}\right)$ normalized such that $\left\|u_{\varepsilon}\right\|_{2}=1$, then, there exists $u_{0} \in H_{0}^{1}([0, \ell])$ and a sequence $\varepsilon_{j} \downarrow 0$ such that $u_{\varepsilon_{j}} \rightarrow u_{0}$ and $u_{0}$ is an eigenfunction of $\left(P_{0}\right)$ associated to $\left(\alpha_{0}, \beta_{0}\right)$.

In the case of periodic homogenization one can do better and obtain an order of convergence. In order to do this one needs to select a point on the curve of the spectrum $\Sigma_{\varepsilon}$ and follow that point as $\varepsilon \downarrow 0$. This is done in the following way: given $t>0$, there exists a unique $\lambda_{k, t, \varepsilon}^{ \pm}$such that $\left(\lambda_{k, t, \varepsilon}^{ \pm}, t \lambda_{k, t, \varepsilon}^{ \pm}\right) \in \mathcal{C}_{k, \varepsilon}^{ \pm}$. Moreover

$$
\mathcal{C}_{k, \varepsilon}^{ \pm}=\bigcup_{t>0}\left\{\left(\lambda_{k, t, \varepsilon}^{ \pm}, t \lambda_{k, t, \varepsilon}^{ \pm}\right)\right\}
$$

Theorem 1.2 (Periodic homogenization). Assume that $m_{\varepsilon}(x)=m\left(\frac{x}{\varepsilon}\right)$ and $n_{\varepsilon}(x)=$ $n\left(\frac{x}{\varepsilon}\right)$ for some $\ell$-periodic functions $m, n \in L^{\infty}(\mathbb{R})$ satisfying (1.1).

Then, we have the bound

$$
\left|\lambda_{k, t, \varepsilon}^{ \pm}-\lambda_{k, t, 0}^{ \pm}\right| \leq C\left(\frac{k}{\ell}\right)^{3} \gamma(t) \varepsilon,
$$

where $C$ depends only on the constants $a, b$ in $(1.1)$ and $\gamma(t)=\max \left\{t^{-\frac{3}{2}}, t^{\frac{1}{2}}\right\}$.

The order of convergence for homogenization of different eigenvalue problems were obtained in $[2,6,8,17]$. Let us recall that in $[2,8]$ the problem was linear, 
and asymptotic expansions were used. On the other hand, in $[6,17]$ the proofs relayed on the variational structure of the problem. Here, there are no variational characterization of the higher curves of the Fučík spectrum, nor linear arguments available, so the proofs are obtained by exploiting the nodal structure of the eigenfunctions.

Organization of the paper. The paper is organized as follows: In Section $\S 2$ we prove the general convergence result, Theorem 1.1, and in Section $\S 3$ we study the periodic oscillation case and prove Theorem 1.2.

\section{A general Convergence Result}

In this section we prove our general convergence result, Theorem 1.1. We begin with an even more general, and therefore more vague, result on the convergence of Fučík eigenvalues.

Throughout this section, we will use the notation $\lambda_{1}^{r, I}$ to denote the first eigenvalue of the Laplacian on the interval $I$ with weight function $r$ complemented with homogeneous Dirichlet boundary conditions. That is, $\lambda_{1}^{r, I}$ is the first eigenvalue of

$$
\begin{cases}-u^{\prime \prime}=\lambda r(x) u & \text { in } I \\ u=0 & \text { on } \partial I\end{cases}
$$

Let us recall, that if the weight $r(x)=$ constant $=c$ then $\lambda_{1}^{r, I}=\lambda_{1}^{c, I}=\frac{\pi^{2}}{c|I|^{2}}$.

Theorem 2.1. Let $m_{\varepsilon}$ and $n_{\varepsilon}$ be two weight functions satisfying (1.1) and assume that $m_{\varepsilon_{j}} \stackrel{*}{\rightarrow} m_{0}, n_{\varepsilon_{j}} \stackrel{*}{\rightarrow} n_{0}$ weakly* in $L^{\infty}([0, \ell])$. Let $\Sigma_{\varepsilon}(\varepsilon \geq 0)$ be the Fučík spectrum given by (1.3).

If $\left(\alpha_{\varepsilon_{j}}, \beta_{\varepsilon_{j}}\right) \in \Sigma_{\varepsilon_{j}}$ are such that $\left(\alpha_{\varepsilon_{j}}, \beta_{\varepsilon_{j}}\right) \rightarrow\left(\alpha_{0}, \beta_{0}\right)$ as $j \rightarrow \infty$, then $\left(\alpha_{0}, \beta_{0}\right) \in$ $\Sigma_{0}$. Moreover, if $u_{\varepsilon_{j}} \in H_{0}^{1}([0, \ell])$ is an eigenfunction of $\left(P_{\varepsilon}\right)$ associated to $\left(\alpha_{\varepsilon_{j}}, \beta_{\varepsilon_{j}}\right)$ normalized such that $\left\|u_{\varepsilon_{j}}\right\|_{2}=1$, then, there exists $u_{0} \in H_{0}^{1}([0, \ell])$ and a subsequence $\varepsilon_{j_{i}} \downarrow 0$ such that $u_{\varepsilon_{j_{i}}} \rightarrow u_{0}$ and $u_{0}$ is an eigenfunction of $\left(P_{0}\right)$ associated to $\left(\alpha_{0}, \beta_{0}\right)$.

Proof. Let $u_{\varepsilon_{j}} \in H_{0}^{1}([0, \ell])$ be an eigenfunction of $\left(P_{\varepsilon}\right)$ associated to $\left(\alpha_{\varepsilon_{j}}, \beta_{\varepsilon_{j}}\right)$ and normalized such that $\left\|u_{\varepsilon_{j}}\right\|_{2}=1$.

Then, since $\left(\alpha_{\varepsilon_{j}}, \beta_{\varepsilon_{j}}\right)$ is bounded and since the weights $m_{\varepsilon_{j}}, n_{\varepsilon_{j}}$ are uniformly bounded, taking $v=u_{\varepsilon_{j}}$ as a test function in (1.2) we get

$$
\begin{aligned}
\int_{0}^{\ell}\left|u_{\varepsilon_{j}}^{\prime}\right|^{2} d x & =\alpha_{\varepsilon_{j}} \int_{0}^{\ell} m_{\varepsilon_{j}}\left(u_{\varepsilon_{j}}^{+}\right)^{2} d x+\beta_{\varepsilon_{j}} \int_{0}^{\ell} n_{\varepsilon_{j}}\left(u_{\varepsilon_{j}}^{-}\right)^{2} d x \\
& \leq C \int_{0}^{\ell}\left(u_{\varepsilon_{j}}^{+}\right)^{2}+\left(u_{\varepsilon_{j}}^{-}\right)^{2} d x=C\left\|u_{\varepsilon_{j}}\right\|_{2}^{2} .
\end{aligned}
$$

Therefore, there exists a subsequence, that we still denoting by $\varepsilon_{j} \downarrow 0$, and $u_{0} \in$ $H_{0}^{1}([0, \ell])$ such that $u_{\varepsilon_{j}} \rightarrow u_{0}$ weakly in $H_{0}^{1}([0, \ell])$ and $u_{\varepsilon_{j}} \rightarrow u_{0}$ uniformly in $[0, \ell]$. These facts automatically imply that $\left(u_{\varepsilon_{j}}^{ \pm}\right)^{2} \rightarrow\left(u_{0}^{ \pm}\right)^{2}$ strongly in $L^{1}([0, \ell])$.

So, we can pass to the limit in the weak form of the equation, (1.2) to obtain

$$
\int_{0}^{\ell} u_{0}^{\prime} v^{\prime} d x=\alpha_{0} \int_{0}^{\ell} m_{0} u_{0}^{+} v d x-\beta_{0} \int_{0}^{\ell} u_{0}^{-} v d x
$$


for every $v \in H_{0}^{1}([0, \ell])$. This finishes the proof.

Let us now see that if we take a sequence $\left\{\left(\alpha_{k, \varepsilon}, \beta_{k, \varepsilon}\right)\right\}_{\varepsilon>0} \subset \mathcal{C}_{k, \varepsilon}$ with a fixed $k \in \mathbb{N}$, then the sequence of eigenvalues remains uniformly bounded as long as they are confined in a cone $\mathcal{K}_{t}$.

Theorem 2.2. Given $0<t<1$ let $\mathcal{K}_{t}$ be the cone defined in (1.5).

Let $k \in \mathbb{N}$ be fixed and consider $\left(\alpha_{k, \varepsilon}, \beta_{k, \varepsilon}\right) \in \mathcal{C}_{k, \varepsilon} \cap \mathcal{K}_{t}$. Then, we have the bound

$$
\max \left\{\alpha_{k, \varepsilon}, \beta_{k, \varepsilon}\right\} \leq t^{-1} \frac{\pi^{2} k^{2}}{a \ell^{2}} .
$$

Proof. Let $u_{k, \varepsilon} \in H_{0}^{1}([0, \ell])$ be a eigenfunction of $\left(P_{\varepsilon}\right)$ associated to $\left(\alpha_{k, \varepsilon}, \beta_{k, \varepsilon}\right) \in$ $\mathcal{C}_{k, \varepsilon} \cap \mathcal{K}_{t}$. Then $u_{k, \varepsilon}$ has exactly $k$ nodal domains. Therefore there exists at least one nodal domain, $I_{\varepsilon}$, such that $\left|I_{\varepsilon}\right| \geq \frac{\ell}{k}$.

Assume that $u_{k, \varepsilon}>0$ in $I_{\varepsilon}$ (the other case can be treated similarly). Therefore, $u_{k, \varepsilon}$ is a weak solution of

$$
\begin{cases}-u_{k, \varepsilon}^{\prime \prime}=\alpha_{k, \varepsilon} m_{\varepsilon} u_{k, \varepsilon} & \text { in } I_{\varepsilon} \\ u_{k, \varepsilon}=0 & \text { on } \partial I_{\varepsilon} .\end{cases}
$$

So, $\alpha_{k, \varepsilon}=\lambda_{1}^{m_{\varepsilon}, I_{\varepsilon}}$. Now, by Sturm's comparison Theorem, we get

$$
\alpha_{k, \varepsilon}=\lambda_{1}^{m_{\varepsilon}, I_{\varepsilon}} \leq \lambda_{1}^{a, I_{\varepsilon}}=\frac{\pi^{2}}{a\left|I_{\varepsilon}\right|^{2}} \leq \frac{\pi^{2} k^{2}}{a \ell^{2}} .
$$

Since $\left(\alpha_{k, \varepsilon}, \beta_{k, \varepsilon}\right) \in \mathcal{K}_{t}$ it follows that,

$$
\beta_{k, \varepsilon} \leq t^{-1} \alpha_{k, \varepsilon}
$$

This completes the proof.

Finally, let us see that the nodal domains of an eigenfunction $u_{k, \varepsilon}$ of $\left(P_{\varepsilon}\right)$ associated to $\left(\alpha_{k, \varepsilon}, \beta_{k, \varepsilon}\right) \in \mathcal{C}_{k, \varepsilon}$ do not degenerate when we pass to the limit $\varepsilon \downarrow 0$ if the eigenvalues $\left(\alpha_{k, \varepsilon}, \beta_{k, \varepsilon}\right)$ are confined to a cone $\mathcal{K}_{t}$.

Theorem 2.3. With the same notations and assumptions of the previous theorem, let $\left(\alpha_{k, \varepsilon}, \beta_{k, \varepsilon}\right) \in \mathcal{C}_{k, \varepsilon} \cap \mathcal{K}_{t}$ and let $u_{k, \varepsilon} \in H_{0}^{1}([0, \ell])$ be an eigenfunction of $\left(P_{\varepsilon}\right)$ associated to $\left(\alpha_{k, \varepsilon}, \beta_{k, \varepsilon}\right)$. Then, every nodal domain $I_{\varepsilon} \subset[0, \ell]$ of $u_{k, \varepsilon}$ verifies the bound

$$
\left|I_{\varepsilon}\right| \geq \frac{\ell}{k} \sqrt{t \frac{a}{b}}
$$

Moreover if we denote by $J_{\varepsilon}$ two consecutive nodal domains, we have the bound

$$
\left|J_{\varepsilon}\right| \geq \frac{\ell}{k} \sqrt{\frac{a}{b}}(1+\sqrt{t})
$$

Proof. Assume that $u_{k, \varepsilon}>0$ in $I_{\varepsilon}$ (the other case is analogous). Arguing as in the proof of Theorem 2.2, we have that $\alpha_{k, \varepsilon}=\lambda_{1}^{m_{\varepsilon}, J_{\varepsilon}}$. So, by Sturm's comparison Theorem,

$$
\alpha_{k, \varepsilon}=\lambda_{1}^{m_{\varepsilon}, J_{\varepsilon}} \geq \lambda_{1}^{b, J_{\varepsilon}}=\frac{\pi^{2}}{b\left|I_{\varepsilon}\right|^{2}} .
$$


Now, using the bound for $\alpha_{k, \varepsilon}$ given in Theorem 2.2, we deduce

$$
\frac{\pi^{2}}{b\left|I_{\varepsilon}\right|^{2}} \leq \alpha_{k, \varepsilon} \leq t^{-1} \frac{\pi^{2} k^{2}}{a \ell^{2}}
$$

and the result follows.

Let now $I_{\varepsilon}^{+}$and $I_{\varepsilon}^{-}$be two consecutive nodal domains, such that $u_{k, \varepsilon}>0$ in $I_{\varepsilon}^{+}$ and $u_{k, \varepsilon}^{-}<0$ in $I_{\varepsilon}^{-}$. We can assume, without loss of generality, that $\alpha_{k, \varepsilon} \leq \beta_{k, \varepsilon}$. Then, from Theorem 2.2, we have that

$$
\alpha_{k, \varepsilon} \leq \frac{\pi^{2} k^{2}}{a \ell^{2}} \quad \text { and } \quad \beta_{k, \varepsilon} \leq t^{-1} \frac{\pi^{2} k^{2}}{a \ell^{2}} .
$$

Then, arguing as in the first part of the proof, we obtain that

$$
\left|I_{\varepsilon}^{+}\right| \geq \frac{\ell}{k} \sqrt{\frac{a}{b}} \text { and }\left|I_{\varepsilon}^{-}\right| \geq \frac{\ell}{k} \sqrt{t \frac{a}{b}}
$$

The result follows observing that $\left|J_{\varepsilon}\right|=\left|I_{\varepsilon}^{+}\right|+\left|I_{\varepsilon}^{-}\right|$.

With the help of Theorems 2.1, 2.2 and 2.3, the proof of Theorem 1.1 follows easily.

Proof of Theorem 1.1. It only remains to see that if $\mathcal{C}_{k, \varepsilon} \ni\left(\alpha_{k, \varepsilon_{j}}, \beta_{k, \varepsilon_{j}}\right) \rightarrow\left(\alpha_{k, 0}, \beta_{k, 0}\right)$ as $j \rightarrow \infty$, then $\left(\alpha_{k, 0}, \beta_{k, 0}\right) \in \mathcal{C}_{k, 0}$. This will follow if we show that an associated eigenfunction $u_{k, 0}$ of $\left(P_{0}\right)$ associated to $\left(\alpha_{k, 0}, \beta_{k, 0}\right)$ has $k$ nodal domains.

But, from Theorem 2.1, we know that $u_{k, \varepsilon_{j}} \rightarrow u_{k, 0}$ weakly in $H_{0}^{1}([0, \ell])$, where $u_{k, \varepsilon_{j}}$ is an eigenfunction of $\left(P_{\varepsilon}\right)$ associated to $\left(\alpha_{k, \varepsilon_{j}}, \beta_{k, \varepsilon_{j}}\right)$ and $u_{k, 0}$ is an eigenfunction of $\left(P_{0}\right)$ associated to $\left(\alpha_{k, 0}, \beta_{k, 0}\right)$. Therefore, we know that $u_{k, 0}$ has only finitely many zeroes and then from Theorem 2.3 we deduce that $u_{k, 0}$ has exactly $k$ nodal domains.

This completes the proof.

\section{An Alternative formulation}

In order to prove the convergence result for periodic homogenization, Theorem 1.2 , it is convenient to consider the following equivalent problem

$$
\left\{\begin{array}{l}
-u^{\prime \prime}=\lambda\left(m(x) u^{+}-\operatorname{tn}(x) u^{-}\right) \quad x \in(0, \ell) \\
u(0)=u(\ell)=0
\end{array}\right.
$$

where $t>0$ is a fixed value. The values of $\lambda \in \mathbb{R}$ for which (3.1) has a nontrivial solution $u$ are called half-eigenvalues, while the corresponding solutions $u$ are called half-eigenfunctions. Problem (3.1) has a positively-homogeneous jumping nonlinearity, and its spectrum is defined as the set

$$
\Sigma_{t}(m, n):=\{\lambda \in \mathbb{R}:(3.1) \text { has non-trivial solution } u\} .
$$

The set $\Sigma_{t}(m, n)$ is divided into two subsets $\Sigma_{t}(m, n)=\Sigma_{t}^{+}(m, n) \cup \Sigma_{t}^{-}(m, n)$ as $\Sigma_{t}^{+}(m, n):=\left\{\lambda \in \Sigma_{t}(m, n): u_{\lambda}^{\prime}(0)>0\right\}, \Sigma_{t}^{-}(m, n):=\left\{\lambda \in \Sigma_{t}(m, n): u_{\lambda}^{\prime}(0)<0\right\}$ where $u_{\lambda}$ is an eigenfunction of (3.1) associated to $\lambda$.

It is shown in [11] that for any $t>0$ both sets $\Sigma_{t}^{ \pm}(m, n)$ consists in a sequence converging to $+\infty$. We denote this sequences by $\left\{\lambda_{k, t}^{ \pm}\right\}_{k \in \mathbb{N}}$. 
Observe that $\lambda_{1, t}^{+}=\lambda_{1}^{m,[0, \ell]}$ and $\lambda_{1, t}^{-}=\lambda_{1}^{t n,[0, \ell]}$. Moreover, each eigenvalue has a unique associated eigenfunction normalized by $\pm u^{\prime}(0)=1$ and the eigenfunction corresponding to $\lambda_{k, t}^{ \pm}$has precisely $k$ nodal domains on $(0, \ell)$, and $k+1$ simple zeros in $[0, \ell]$. See $[11]$ for a proof of these facts.

We can rewrite problems $\left(P_{\varepsilon}\right)$ and $\left(P_{0}\right)$ by taking $\lambda_{\varepsilon}=\alpha_{\varepsilon}$ and $\beta_{\varepsilon}=t \alpha_{\varepsilon}$, and we obtain the following problems

$$
\left\{\begin{array}{l}
-u^{\prime \prime}=\lambda\left(m_{\varepsilon}(x) u^{+}-\operatorname{tn}_{\varepsilon}(x) u^{-}\right) \quad x \in(0, \ell) \\
u(0)=u(\ell)=0,
\end{array}\right.
$$

for $\varepsilon \geq 0$.

We denote the eigenvalues of $\left(P_{\varepsilon}^{t}\right)$ by $\left\{\lambda_{k, t, \varepsilon}^{ \pm}\right\}_{k \in \mathbb{N}}$.

Now, Theorem 1.1 trivially implies the following

Theorem 3.1. Let $\left\{m_{\varepsilon}\right\}_{\varepsilon>0}$ and $\left\{n_{\varepsilon}\right\}_{\varepsilon>0}$ be two families of weights in $L^{\infty}([0, \ell])$ satisfying (1.1). Assume, moreover that $m_{\varepsilon} \stackrel{*}{\rightarrow} m_{0}$ and $n_{\varepsilon} \stackrel{*}{\rightarrow} n_{0}$ weakly ${ }^{*}$ in $L^{\infty}([0, \ell])$ for some limit weights $m_{0}$ and $n_{0}$.

Let us denote by $\left\{\lambda_{k, t, \varepsilon}^{ \pm}\right\}_{k \in \mathbb{N}}$ the eigenvalues of $\left(P_{\varepsilon}^{t}\right)$ for $\varepsilon \geq 0$. Then

$$
\lim _{\varepsilon \rightarrow 0} \lambda_{k, t, \varepsilon}^{ \pm}=\lambda_{k, t, 0}^{ \pm}
$$

Now we specialize to the periodic case, and obtain the following refinement.

Theorem 3.2. In addition to the assumptions of Theorem 3.1, assume that $m_{\varepsilon}(x)=$ $m\left(\frac{x}{\varepsilon}\right)$ and $n_{\varepsilon}(x)=n\left(\frac{x}{\varepsilon}\right)$ for some $\ell$-periodic functions $m, n \in L^{\infty}(\mathbb{R})$. Then, for $0<t<1$,

$$
\left|\lambda_{k, t, \varepsilon}^{ \pm}-\lambda_{k, t, 0}^{ \pm}\right| \leq C\left(\frac{k}{\ell}\right)^{3} t^{-\frac{3}{2}} \varepsilon
$$

where $C$ depends onlyt on $a, b$ in (1.1).

Observe that Theorem 1.2 follows directly from Theorem 3.2. In fact, Theorem 3.2 is Theorem 1.2 in the case $0<t<1$ and the case where $t>1$ follows from this one by symmetry. To be precise, if $t>1$ and $u_{\varepsilon}$ is an eigenfunction associated to $\lambda_{k, t, \varepsilon}^{ \pm}$, we denote $v_{\varepsilon}=-u_{\varepsilon}$ and so it verifies

$$
\left\{\begin{array}{l}
-v_{\varepsilon}^{\prime \prime}=t \lambda_{k, t, \varepsilon}^{ \pm}\left(n_{\varepsilon} v_{\varepsilon}^{+}-t^{-1} m_{\varepsilon} v_{\varepsilon}^{-}\right) \quad \text { in }(0, \ell) \\
v(0)=v(\ell)=0 .
\end{array}\right.
$$

Therefore, from Theorem 3.2 we have the estimate

$$
\left|t \lambda_{k, t, \varepsilon}^{ \pm}-t \lambda_{k, t, 0}^{ \pm}\right| \leq C\left(\frac{k}{\ell}\right)^{3} t^{\frac{3}{2}} \varepsilon,
$$

and Theorem 1.2 follows directly from this former inequality.

For the proof of Theorem 3.2, we need the order of convergence of the nodal domains of the eigenfunctions. We will perform this task in a series of lemmas.

Lemma 3.3. Let $\left(\lambda_{k, t, \varepsilon}^{ \pm}, u_{k, t, \varepsilon}\right)$ be an eigenpair of $\left(P_{\varepsilon}^{t}\right)$. We denote by $\left\{I_{j, \varepsilon}^{+}\right\}_{j} \cup$ $\left\{I_{i, \varepsilon}^{-}\right\}_{i}$ the nodal domains of $u_{k, t, \varepsilon}$, that is each $I_{l, \varepsilon}^{ \pm}$is an open connected, pairwise disjoint intervals, such that

$$
[0, \ell]=\bigcup_{j} \overline{I_{j, \varepsilon}^{+}} \cup \bigcup_{i} \overline{I_{i, \varepsilon}^{-}}
$$


and $u_{k, t, \varepsilon}>0$ on $I_{j, \varepsilon}^{+}, u_{k, t, \varepsilon}<0$ on $I_{i, \varepsilon}^{-}$.

Then, ||$I_{j, \varepsilon}^{+}|-| I_{l, \varepsilon}^{+}||<2 \varepsilon$ and ||$I_{i, \varepsilon}^{-}|-| I_{l, \varepsilon}^{-}||<2 \varepsilon$

Proof. We make the proof for the positive nodal domains $\left\{I_{j, \varepsilon}^{+}\right\}_{j}$. The other one is analogous.

First, let $j_{0}$ be such that $\left|I_{j_{0}, \varepsilon}^{+}\right| \leq\left|I_{j, \varepsilon}^{+}\right|$for any $j$.

Assume that there exists $j$ such that $\left|I_{j, \varepsilon}^{+}\right|>\left|I_{j_{0}, \varepsilon}^{+}\right|+2 \varepsilon$. Then, there exists an integer $h \in \mathbb{Z}$ such that $h \varepsilon+I_{j_{0}, \varepsilon}^{+} \subset I_{j, \varepsilon}^{+}$.

Now, if we denote

$$
v_{\varepsilon}(x)= \begin{cases}u_{k, t, \varepsilon}(x-h \varepsilon) & \text { if } x \in I_{j_{0}, \varepsilon}^{+}+h \varepsilon \\ 0 & \text { elsewhere }\end{cases}
$$

then $v_{\varepsilon} \in H_{0}^{1}\left(I_{j, \varepsilon}^{+}\right)$, and so

$$
\begin{aligned}
\lambda_{k, t, \varepsilon}^{+}=\lambda_{1}^{m_{\varepsilon}, I_{j, \varepsilon}^{+}} & =\inf _{v \in H_{0}^{1}\left(I_{j, \varepsilon}^{+}\right)} \frac{\int_{I_{j, \varepsilon}^{+}}\left(v^{\prime}\right)^{2} d x}{\int_{I_{j, \varepsilon}^{+}} m_{\varepsilon} v^{2} d x} \\
& \leq \frac{\int_{I_{j, \varepsilon}^{+}}\left(v_{\varepsilon}^{\prime}\right)^{2} d x}{\int_{I_{j, \varepsilon}^{+}} m_{\varepsilon} v_{\varepsilon}^{2} d x} \\
& =\frac{\int_{I_{0}, \varepsilon}^{+}\left(u_{k, t, \varepsilon}^{\prime}\right)^{2} d x}{\int_{I_{j_{0}, \varepsilon}^{+}} m_{\varepsilon} u_{k, t, \varepsilon}^{2} d x} \\
& =\lambda_{k, t, \varepsilon}^{+},
\end{aligned}
$$

where we have used the periodicity of the weight $m_{\varepsilon}$. This shows that $v_{\varepsilon}$ is an eigenfunction associated to $\lambda_{1}^{m_{\varepsilon}, I_{j, \varepsilon}^{+}}$, but this is a contradiction since $v_{\varepsilon}$ vanishes in a set of positive measure.

The proof is complete.

The following elementary lemma will be most useful.

Lemma 3.4. Let $M \in \mathbb{R}$ and $K \in \mathbb{N}$. Assume that for every $\varepsilon>0$, there exists $\left\{a_{i}^{\varepsilon}\right\}_{i=1}^{K} \subset \mathbb{R}$, such that

$$
\sum_{i=1}^{K} a_{i}^{\varepsilon}=M \quad \text { and } \quad\left|a_{i}^{\varepsilon}-a_{j}^{\varepsilon}\right|<\varepsilon, \text { for every } 1 \leq i, j \leq K .
$$

Then

$$
\left|a_{i}^{\varepsilon}-\frac{M}{K}\right|<\varepsilon, \text { for every } 1 \leq i \leq K
$$

Proof. Let $i_{0}=i_{0}(\varepsilon) \in\{1, \ldots, K\}$ be such that $a_{i_{0}}^{\varepsilon} \leq a_{i}^{\varepsilon}$ for every $1 \leq i \leq K$. Then

$$
K a_{i_{0}}^{\varepsilon} \leq \sum_{i=1}^{K} a_{i}^{\varepsilon}=M
$$


and so $a_{i_{0}}^{\varepsilon} \leq \frac{M}{K}$. Therefore, for any $1 \leq i \leq K$,

$$
a_{i}^{\varepsilon}<a_{i_{0}}^{\varepsilon}+\varepsilon \leq \frac{M}{K}+\varepsilon .
$$

On the other hand, if we now take $i_{1}=i_{1}(\varepsilon) \in\{1, \ldots, K\}$ such that $a_{i_{1}}^{\varepsilon} \geq a_{i}^{\varepsilon}$ for every $1 \leq i \leq K$, then

$$
K a_{i_{i}}^{\varepsilon} \geq \sum_{i=1}^{K} a_{i}^{\varepsilon}=M
$$

thus $a_{i_{1}}^{\varepsilon} \geq \frac{M}{K}$. Hence, for any $1 \leq i \leq K$,

$$
a_{i}^{\varepsilon} \geq a_{i_{1}}^{\varepsilon}-\varepsilon \geq \frac{M}{K}-\varepsilon .
$$

The lemma is proved.

Lemma 3.3 together with Lemma 3.4 imply the following corollary.

Corollary 3.5. Let $\left(\lambda_{k, t, \varepsilon}^{ \pm}, u_{k, t, \varepsilon}\right)$ be an eigenpair of $\left(P_{\varepsilon}^{t}\right)$. We denote by $\left\{I_{j, \varepsilon}^{+}\right\}_{j} \cup$ $\left\{I_{i, \varepsilon}^{-}\right\}_{i}$ the nodal domains of $u_{k, t, \varepsilon}$. Then

$$
|| I_{j, \varepsilon}^{+} \cup I_{j, \varepsilon}^{-}\left|-\frac{2 \ell}{k}\right| \leq 4 \varepsilon \text {. }
$$

Proof. Assume first that $k$ is even. So $k=2 n$ for some $n \in \mathbb{N}$. Then, the number of positive nodal domains equals the number of negative nodal domains and both equal $n$. Therefore

$$
\ell=\sum_{j=1}^{n}\left|I_{j, \varepsilon}^{+}\right|+\sum_{j=1}^{n}\left|I_{j, \varepsilon}^{-}\right|=\sum_{j=1}^{n}\left|I_{j, \varepsilon}^{+} \cup I_{j, \varepsilon}^{-}\right| .
$$

Let us call $a_{j}^{\varepsilon}=\left|I_{j, \varepsilon}^{+} \cup I_{j, \varepsilon}^{-}\right|$, and by Lemma 3.3 we have that $\left|a_{j}^{\varepsilon}-a_{i}^{\varepsilon}\right|<4 \varepsilon$. Hence, we can invoke Lemma 3.4 and conclude the desired result.

If now $k$ is odd, we consider the problem in $[-\ell, \ell]$ and extend $u_{k, t, \varepsilon}$ by odd reflexion and so we end up with a even number of positive and negative nodal domains. We apply the first part of the proof and from that the result follows. We leave the details to the reader.

Remark 3.6. Observe that, since $m_{0}$ and $n_{0}$ are constant functions, it holds that $\left|I_{j, 0}^{+} \cup I_{j, 0}^{-}\right|=\frac{2 \ell}{k}$. See [7].

The other key ingredient in the proof of Theorem 3.2 is the following result due to [17], recently improved in [18].

Theorem 3.7 ([18], Theorem 1.2). Under the above assumptions and notations, it holds that

$$
\left|\lambda_{2, t, \varepsilon}^{ \pm}-\lambda_{2, t, 0}^{ \pm}\right| \leq C \varepsilon t^{-\frac{3}{2}}
$$

where $C$ is a constant depending only on the bounds $a, b$ in (1.1).

Remark 3.8. In [17] the obtained bound is slightly worse than (3.4). In fact, is was proved in [17, Theorem 4.2] that

$$
\left|\lambda_{2, t, \varepsilon}^{ \pm}-\lambda_{2, t, 0}^{ \pm}\right| \leq C^{\prime} \varepsilon t^{-2}
$$

with $C^{\prime}$ depending also on $a, b$. 
With all of these preliminaries, we can now prove the main result of the section.

Proof of Theorem 3.2. Let $u_{k, t, \varepsilon}$ be an eigenfunction of $\left(P_{\varepsilon}^{t}\right)$ associated to $\lambda_{k, t, \varepsilon}^{+}$, for $\varepsilon \geq 0$. The case of $\lambda_{k, t, \varepsilon}^{-}$is completely analogous.

Let $J_{\varepsilon}=I_{1, \varepsilon}^{+} \cup I_{1, \varepsilon}^{-}$be the union of the first two nodal domains of $u_{k, t, \varepsilon}$. Let us denote $J_{\varepsilon}=\left(0, c_{\varepsilon}\right)$. Observe that $u_{k, t, \varepsilon}>0$ in $I_{\varepsilon}$ for $\varepsilon \geq 0$ and that, by Theorem 2.3 , we have the bound

$$
c_{\varepsilon} \geq \frac{\ell}{k} \sqrt{\frac{a}{b}}(1+\sqrt{t}) .
$$

Arguing as in Theorem 2.2, we deduce that for any $\varepsilon \geq 0$,

$$
\lambda_{k, t, \varepsilon}^{+}=\lambda_{2}^{m_{\varepsilon}, t n_{\varepsilon}, J_{\varepsilon}}
$$

where $\lambda_{2}^{m_{\varepsilon}, t n_{\varepsilon}, J_{\varepsilon}}$ is the second eigenvalue of $\left(P_{\varepsilon}^{t}\right)$ in the interval $J_{\varepsilon}$.

Performing a change of variables is easy to see that

$$
c_{\varepsilon}^{2} \lambda_{2}^{m_{\varepsilon}, t n_{\varepsilon}, J_{\varepsilon}}=\lambda_{2}^{m_{\varepsilon^{\prime}}, t n_{\varepsilon^{\prime}},[0,1]},
$$

where $\varepsilon^{\prime}=\frac{\varepsilon}{c_{\varepsilon}}$. Observe that from (3.5) it follows that $\varepsilon^{\prime} \rightarrow 0$.

Now, using Theorem 3.7 we obtain that

$$
\left|\lambda_{2}^{m_{\varepsilon^{\prime}}, t n_{\varepsilon^{\prime},[0,1]}}-\lambda_{2}^{m_{0}, t n_{0},[0,1]}\right| \leq C \varepsilon^{\prime} t^{-\frac{3}{2}} \leq C \frac{k}{\ell} t^{-\frac{3}{2}} \varepsilon .
$$

where $C$ depends on the constants $a, b$ in (1.1).

Therefore, by(3.5), (3.7) and (3.8), we find

$$
\begin{aligned}
\left|\lambda_{k, t, \varepsilon}^{+}-\lambda_{k, t, 0}^{+}\right| & =\left|c_{\varepsilon}^{-2} \lambda_{2}^{m_{\varepsilon^{\prime}}, t n_{\varepsilon^{\prime}},[0,1]}-c_{0}^{-2} \lambda_{2}^{m_{0}, t n_{0},[0,1]}\right| \\
& \leq c_{\varepsilon}^{-2}\left|\lambda_{2}^{m_{\varepsilon^{\prime}}, t n_{\varepsilon^{\prime}},[0,1]}-\lambda_{2}^{m_{0}, t n_{0},[0,1]}\right|+\lambda_{2}^{m_{0}, t n_{0},[0,1]}\left|c_{\varepsilon}^{-2}-c_{0}^{-2}\right| \\
& \leq C\left(\frac{k}{\ell}\right)^{3} t^{-\frac{3}{2}} \varepsilon+\lambda_{2}^{m_{0}, t n_{0},[0,1]}\left|c_{\varepsilon}^{-2}-c_{0}^{-2}\right| .
\end{aligned}
$$

Now, from Corollary 3.5 and the subsequent remark,

$$
\left|c_{\varepsilon}^{-2}-c_{0}^{-2}\right| \leq C\left(\frac{k}{\ell}\right)^{3} \varepsilon
$$

with $C$ a universal constant.

Finally, we observe that from Theorem 2.3,

$$
\lambda_{2}^{m_{0}, t n_{0},[0,1]}=\lambda_{1}^{m_{0}, I_{1, \varepsilon}^{+}}=\frac{\pi^{2}}{m_{0}\left|I_{1, \varepsilon}^{+}\right|^{2}} \leq C t,
$$

with $C$ depending on $a, b$ in (1.1).

\section{ACKNOWLEDGEMENTS}

This paper was partially supported by Universidad de Buenos Aires under grant UBACyT 20020130100283BA and by ANPCyT under grant PICT 2012-0153. The authors are members of CONICET. 


\section{REFERENCES}

1. L. Baffico, C. Conca, and M. Rajesh, Homogenization of a class of nonlinear eigenvalue problems, Proc. Roy. Soc. Edinburgh Sect. A 136 (2006), no. 1, 7-22. MR 2217505 (2007b:35021)

2. Carlos Castro and Enrique Zuazua, Low frequency asymptotic analysis of a string with rapidly oscillating density, SIAM J. Appl. Math. 60 (2000), no. 4, 1205-1233 (electronic). MR 1760033 (2001h:34117)

3. Valeria Chiadò Piat, Gianni Dal Maso, and Anneliese Defranceschi, G-convergence of monotone operators, Ann. Inst. H. Poincaré Anal. Non Linéaire 7 (1990), no. 3, 123-160. MR 1065871 (91f:49018)

4. E. N. Dancer, On the Dirichlet problem for weakly non-linear elliptic partial differential equations, Proc. Roy. Soc. Edinburgh Sect. A 76 (1976/77), no. 4, 283-300. MR 0499709 (58 \#17506)

5. Julián Fernández Bonder, Juan P. Pinasco, and Ariel M. Salort, Convergence rate for some quasilinear eigenvalues homogenization problems, J. Math. Anal. Appl. 423 (2015), no. 2, 1427-1447. MR 3278207

6. - Quasilinear eigenvalues, Rev. Un. Mat. Argentina 56 (2015), no. 1, 1-25. MR 3361839

7. Svatopluk Fučík and Alois Kufner, Nonlinear differential equations, Studies in Applied Mechanics, vol. 2, Elsevier Scientific Publishing Co., Amsterdam-New York, 1980. MR 558764 (81e:35001)

8. Carlos E. Kenig, Fanghua Lin, and Zhongwei Shen, Convergence rates in $L^{2}$ for elliptic homogenization problems, Arch. Ration. Mech. Anal. 203 (2012), no. 3, 1009-1036. MR 2928140

9. Srinivasan Kesavan, Homogenization of elliptic eigenvalue problems. I, Appl. Math. Optim. 5 (1979), no. 2, 153-167. MR 533617 (80f:65111)

10. _ Homogenization of elliptic eigenvalue problems. II, Appl. Math. Optim. 5 (1979), no. 3, 197-216. MR 546068 (80i:65110)

11. Wei Li and Ping Yan, Various half-eigenvalues of scalar p-Laplacian with indefinite integrable weights, Abstr. Appl. Anal. (2009), Art. ID 109757, 27. MR 2539910 (2010g:34205)

12. _ Continuity and continuous differentiability of half-eigenvalues in potentials, Commun. Contemp. Math. 12 (2010), no. 6, 977-996. MR 2748281 (2012c:34292)

13. Josef Malík, Oscillations in cable-stayed bridges: existence, uniqueness, and homogenization of cable systems, J. Math. Anal. Appl. 266 (2002), no. 1, 100-126. MR 1876772 (2002j:74030)

14. O. A. Olernik, A. S. Shamaev, and G. A. Yosifian, Mathematical problems in elasticity and homogenization, Studies in Mathematics and its Applications, vol. 26, North-Holland Publishing Co., Amsterdam, 1992. MR 1195131 (93k:35025)

15. Juan P. Pinasco and Ariel M. Salort, Asymptotic behavior of the curves in the fucik spectrum, Commun. Contemp. Math. to appear (2015).

16. Bryan P. Rynne, The Fučí spectrum of general Sturm-Liouville problems, J. Differential Equations 161 (2000), no. 1, 87-109. MR 1740358 (2000j:34031)

17. Ariel Martin Salort, Convergence rates in a weighted Fučik problem, Adv. Nonlinear Stud. 14 (2014), no. 2, 427-443. MR 3194363

18. _ Homogenization of Fučik eigenvalues by optimal partition methods, preprint (2016), $13 p$.

Departamento de Matemática, FCEyn - Universidad de Buenos Aires and

IMAS - CONICET

Ciudad Universitaria, Pabellón I (1428) Av. Cantilo s/N.

Buenos Aires, Argentina.

E-mail address, J. Fernández Bonder: jfbonder@dm.uba.ar

URL: http://mate.dm.uba.ar/ jfbonder

E-mail address, J.P. Pinasco: jpinasco@dm.uba.ar

URL: http://mate.dm.uba.ar/ jpinasco

E-mail address, A.M. Salort: asalort@dm.uba.ar

$U R L:$ http://mate.dm.uba.ar/ asalort 